\title{
Die tierärztliche Dokumentationspflicht und das Einsichtsrecht in tierärztliche Behandlungsunterlagen
}

\author{
Kai Bemmann \\ Kanzlei Berner, Fischer und Partner, Verden
}

\section{Vorwort}

Die Tiermedizin ist eine Naturwissenschaft, die erhebliches handwerkliches Geschick und technisches Verständnis erfordert. Die Schriftstellerei ist keine Naturwissenschaft, geschweige denn erfordert sie die beiden weiteren vorstehend genannten Fähigkeiten. Wohl gerade deshalb ist sie regelmäßig nicht die Lieblingstätigkeit eines Tiermediziners und erst recht nicht des praktischen Tierarztes. So wird dann die tierärztliche Dokumentation oft als notwendiges Übel und lästige Pflicht, aber weniger als sinnvolle tiermedizinische Tätigkeit angesehen. Das kann sich allerdings insbesondere in der Pferdemedizin als Fehleinschätzung erweisen. Denn durch den Bedeutungswandel des Pferdes vom landwirtschaftlichen, industriellen und militärischen Arbeits- und Nutztier zum wertvollen Hochleistungssportler und zur hochpreisigen Handelsware hat sich auch das Verhältnis der Pferdeeigentümer zum behandelnden Tierarzt verändert (Schüle 2003). Die Klientel des Tierarztes ist nicht nur generell medizinkritischer geworden. Sie rekrutiert sich auch aus anderen Kreisen der Bevölkerung, die weniger angeborenes oder erlerntes Verständnis für die Natur und die Tiermedizin aufbringen, dafür aber höhere Erwartungshaltungen entfalten und insbesondere prozessfreudiger sind. Ein deutliches Zeichen dafür sind die vielen Tierarzt-Haftpflichtrechtsstreitigkeiten, obwohl die Haftungssituation des Tierarztes sich nach der Schuldrechtsreform insbesondere im Bereich der Kaufuntersuchungen deutlich entschärft haben müsste. Denn nach dem Wegfall der viehkaufrechtlichen Sonderregelungen im BGB und der Viehmängelverordnung stehen dem Pferdekäufer breit gefächerte Möglichkeiten zur Verfügung, den Pferdeverkäufer in die Sachmängelhaftung zu nehmen. Folglich muss der bis zur Schuldrechtsreform häufig unternommene Versuch, die nicht vorhandenen kaufvertraglichen Gewährleistungsmöglichkeiten über eine Haftung des kaufuntersuchenden Tierarztes zu kompensieren, nicht mehr unternommen werden.

In der täglichen Praxis steht der häufig allein und ambulant behandelnde praktische Tierarzt oftmals mehreren Personen aus der Sphäre des Pferdeeigentümers - nämlich dessen Bereiter und Pferdepfleger und zumeist auch noch dessen Familienangehörigen - gegenüber. Diese Personen sind dann in einem Rechtsstreit regelmäßig die vom Pferdeeigentümer als Beweismittel benannten Zeugen für ein angeblich kunstfehlerhaftes Vorgehen des Tierarztes. Dem kann der Tierarzt wenig entgegensetzen, wenn er nicht auf eine pflichtgemäß geführte Dokumentation in seinen tierärztlichen Behandlungsunterlagen zurückgreifen kann. Die Dokumentation steht ihm im Tie- rarzthaftungsrechtsstreit nämlich als ideales Beweismittel zur Verfügung und plötzlich kann sich die ungeliebte Schreibarbeit als "schärfstes Schwert" des Tierarztes im Haftungsrechtsstreit erweisen. Die Dokumentation ist aber nicht nur eine geeignete Absicherung gegen juristische Nachteile, sondern auch eine gute Möglichkeit, Qualität in der Tiermedizin und Kommunikation mit den Patienteneigentümern zu demonstrieren (Mehrhoff 1990). Dies macht es sinnvoll, sich mit den Krankenunterlagen, deren Rechtsnatur sowie der Dokumentationspflicht und ihren Auswirkungen näher zu befassen.

\section{Begriff und Rechtscharakter der Dokumentation}

Unter der tierärztlichen Dokumentation werden alle vom Tierarzł getätigten Aufzeichnungen verstanden. Hierunter fallen nicht nur die vom Tierarzt und/oder seinen Mitarbeitern gefertigten Schriftstücke wie Krankengeschichte, Karteikarten, Tagebuch, Rechnungen, Tierarztbriefe, Überweisungsschreiben, Operations- und Laborberichte sowie Protokolle. Vielmehr gehören zur Dokumentation auch Präparate, Röntgen- und Ultraschallbilder und technische Aufzeichnungen (Jäckel 1971; Wasserburg 1980). Die möglichen Gegenstände und Mittel der Dokumentation dürfen keineswegs abschließend, sondern nur beispielhaft verstanden werden. Neve tiermedizinische Erkenntnismethoden können durchaus zusätzliche Arten der Dokumentation begründen (Bender 1997).

Die schriftlichen Aufzeichnungen des Tierarztes stellen die Verkörperung einer Gedankenäußerung dar und sind deshalb Urkunden im prozessualen Sinne gem. §§ 415 ff ZPO, 249 StPO. Dies gilt nicht für Röntgenbilder, Ultraschallbilder, Szintigramme oder ähnliche Dokumentationen. Hierbei handelt es sich um technische Aufzeichnungen im Sinne von $\S \S 268$, 274 StGB. Der wesentliche Unterschied besteht in der verschiedenen Behandlung im Prozess- und materiellen Recht. Urkunden können als Beweismittel Gegenstand eines Vorlegungsantrages im Zivilprozess gem. § 421 ZPO sein. Im Fall der Nichtvorlage der Urkunde entstehen prozessuale Nachteile für den Vorlegungspflichtigen im Sinne von § 427 ZPO. Es kann nämlich der vom Antragsteller unter Bezugnahme auf die im Vorlegungsantrag genannte Urkunde gehaltene Vortrag als richtig unterstellt werden. Währenddessen können die anderen Dokumentationen (nur) Gegenstand richterlichen Augenscheines oder einer sachverständigen Begutachtung sein. Darüber hinaus können tiermedizinische Dokumentationen unter bestimmten, im Folgenden noch zu erörternden 
Voraussetzungen, Urkunden im Sinne von $\S \S 810,811$ BGB und deshalb Gegenstand eines selbständigen materiellrechtlichen Einsichtsanspruchs außerhalb eines Rechtsstreits sein.

Den technischen Aufzeichnungen und den Urkunden ist gemeinsam, dass sie im Falle der Fälschung strafrechtliche Bedeutung gem. $\S \S 267$ ff. StGB für den jeweiligen Täter erlangen. Außerdem stehen sowohl Urkunden als auch technische Aufzeichnungen sowie alle anderen denkbaren Dokumentationen im Eigentum des Tierarztes als Hersteller. Der Tierarzt enwirbt Eigentum an seiner Dokumentation gem. § 950 BGB. Dies verliert er auch nicht durch die Honorarzahlung des Auftraggebers. Durch die Honorarzahlung wird lediglich die tierärztliche Dienstleistung abgegolten, die u. a. durch das Dokumentieren erbracht wird. Der Tiereigentümer erwirbt aber kein Eigentum und dementsprechend keinen Herausgabeanspruch an der Dokumentation. Ebenso bleibt der Tierarzt Urheber (BGH 1952). Dadurch ist der Tierarzt auch noch als geistiger Schöpfer seiner Dokumentation geschützt und verliert diesen Schutz nicht dadurch, dass er zum Zeitpunkt der Herstellung der Dokumentation in einem Dienstverhältnis zu einem Dritten stand. Sofern mehrere Tierärzte die Dokumentation erstellen, werden diese gemeinschaftliche Eigentümer der Dokumentation und ihnen stehen die Urheberrechte gemeinsam zu.

\section{Die vertragliche Verpflichtung zur Dokumentation}

Ähnlich wie die Aufklärung ist die Dokumentation keine originäre Pflicht des medizinischen Behandlungsvertrages. Sie hat sich vielmehr erst langsam entwickelt. Auch diese Entwick-lung nahm ihren Anfang in der Humanmedizin. Dort erlangte die Dokumentation im Lichte der Grundrechte des Patienten wesentlich schneller und auf andere Weise eine Bedeutung als in der Tiermedizin. Gleichwohl wurde die Dokumentation auch in der Humanmedizin lange Jahre nur als eine freiwillige Leistung des Arztes verstanden, die er in seinem eigenen Interesse erbringt und an der ein Patient keinerlei Rechte hat (Wiethaupt 1954). Sie diente vornehmlich als Gedächtnisstütze des behandelnden Arztes (Jäckel 1971). Der Arzt leistete die Dokumentation freiwillig, einer ärztlichen Gepflogenheit entsprechend (BGH 1972).

Der "gute ärztliche Brauch" und die standesrechtliche Pflicht zur Dokumentation sind insbesondere im Spannungsfeld zwischen den Grundrechten des Patienten als Mensch gem. Art. 1, 2 GG und der aus dem Grundrecht der Berufs- und Wissenschaftsfreiheit gem. Art. 12, 5 GG abgeleiteten Therapiefreiheit des Arztes zu untersuchen. Der Patient kann durch den Behandlungsauftrag nur bestimmen, dass er behandelt wird. Aufgrund der ärztlichen Therapiefreiheit hat der Patient regelmäßig keinen Einfluss darauf, wie er behandelt wird. Deshalb war an der Freiwilligkeit der Dokumentation im Sinne eines "guten ärztlichen Brauchs" zum alleinigen Zweck der Gedächtnisunterstützung des behandelnden Arztes Kritik laut geworden. Andererseits wurde von Seiten der ärztlichen Interessenvertreter eingewandt, dass eine Verpflichtung zur Dokumentation für den Arzt die Gefahr nach sich ziehe, dass dieser in einem gegen ihn aufgrund einer Patientenanzeige eingeleiteten Strafverfahren Beweismittel zu seinen Lasten schaffe, wodurch der auch ihm zustehende Grund- und strafrechtliche Schutz vor Selbstbezichtigung gefährdet und durch eine Beschlagnahme der Dokumentation sein Schweigerecht als Beschuldigter unterlaufen werde. Dieses Argument hat jedoch nur auf den ersten Blick schweres Gewicht; denn es darf nicht zur Beseitigung der eingegangen schuldrechtlichen Verpflichtungen führen. Dies war und ist bereits anlässlich anderer, bekannter Fallkonstellationen hinreichend geklärt. So ist z. B. jeder Kfz-Halter verpflichtet, seinem Versicherer gegenüber wahre und vollständige Angaben zu einem Unfall, dem Tatfahrer und gegebenenfalls dessen Alkoholisierung zu machen und es ist inzwischen unstreitig, dass im Verdachtsfalle der Schadensbericht beim $\mathrm{Kfz}$-Versicherer beschlagnahmt und mit seiner Hilfe ein Tatfahrer überführt werden kann. Deshalb steht es außer Frage, dass die Gesichtspunkte des strafprozessualen Schweigerechts dann zurückzutreten haben, wenn es zu den Dienstpflichten des ärztlichen Behandlungsvertrages gehört, eine Dokumentation vorzunehmen. Würden solche Einwendungen gegen eine Dokumentationspflicht durchgreifen, wäre eine vertravensvolle ärztliche Tätigkeit nicht mehr denkbar (Bender 1997). Deshalb erfordert es das Gebot einer Gleichberechtigung der Parteien eines ärztlichen Behandlungsvertrages, dem Privileg der ärztlichen Therapiefreiheit und dem überlegenen Fachwissen die vertragliche Verpflichtung zur Herstellung einer Dokumentation entgegenzusetzen, die auch im Interesse des Patienten zu erstellen ist.

Im Lichte der vorgestehenden Gedanken wandelte sich die Rechtsprechung. Der einerseits selbstverständlich, aber andererseits im ausschließlichen Eigeninteresse gepflegte "gute ärztliche Brauch" der Dokumentation erwuchs zu einer vertraglichen Nebenpflicht des Behandlungsvertrages, die es auch im Interesse des Patienten zu erfüllen gilt. Dies ist seit der Grundsatzentscheidung des BGH (BGH 1978 a) ständige Rechtsprechung und herrschende Meinung in der Literatur.

Die Dokumentationspflicht entsteht sukzessive mit den einzelnen Behandlungsabschnitten. Keinesfalls beginnt sie erst nach Abschluss einer Behandlung oder Therapie oder gar erst nachträglich im Hinblick auf einen Prozess. Dies würde nämlich dem Sinn einer medizinischen Dokumentation zuwiderlaufen. Die Gefahr einer ungenaven und unrichtigen Dokumentation wäre dadurch eröffnet (Wasserburg 1980). Vielmehr muss die Dokumentationspflicht insbesondere drei Anforderungen genügen. Sie muss zur Behandlungsunterstützung, Behandlungsinformation und Beweissicherung geeignet sein (Bender 1997). Vollständigkeit, Wahrheit und Klarheit sind dabei die Kriterien, die der Arzł im Rahmen einer ordnungsgemäßen Dokumentation zu beachten hat. Dies gilt insbesondere auch deshalb, weil der vornehmliche Sinn einer Dokumentationspflicht nicht in der Hilfestellung bei der Realisierung von Schadenersatzansprüchen des Patienten besteht, sondern der Patient über seinen gesundheitlichen Zustand aufgeklärt wird. Außerdem hilft die Dokumentation anderen Ärzten bei der Weiterbehandlung. Letztendlich ist sie dem Patienten eine Entscheidungshilfe über die Art und Notwendigkeit einer Weiterbehandlung (Wasserburg 1980).

Unter prozessualen Gesichtspunkten dient sie zur Gewährleistung einer gleichwertigen Stellung von Arzt und Patient im Rechtsstreit. Die Gleichwertigkeit ist zwingend herzustellen, weil die Waffengleichheit ein Prozessgrundrecht darstell (BVerfG 1979). Diesem Grundrecht wird maßgeblich durch die Auferlegung einer auch im Interesse des Patienten einzuhaltenden Verpflichtung zur Dokumentation ärztlicher Behandlungen Rechnung getragen (BGH 1975; BGH 1978 b; Franzki und Franzki 1975). 


\section{Die gesetzliche Verpflichtung zur Dokumentation und Aufbewahrungsfristen}

Neben der durch den Behandlungsvertrag begründeten Pflicht zur Dokumentation gibt es einige gesetzliche Pflichten zur Dokumentation und Aufbewahrung z. B. gem. § 147 I AO, § 42 StrSchV sowie gem. § 28 RöV (Strunz 1990). Insbesondere die in der RöV vorgeschriebene Dokumentationsverpflichtung erlangt auch in der Tiermedizin erhebliche Bedeutung.

Abgesehen von den wenigen gesetzlichen Dokumentationsverpflichtungen und der vertraglichen Nebenpflicht aus dem Behandlungsvertrag ergibt sich aus den Berufsordnungen der Tierärztekammern der einzelnen Bundesländer eine Standespflicht zur Erstellung einer Dokumentation. Die Berufsordnungen der Bundesländer haben sinngleiche Formulierungen. So ist gem. $\S 2$ Abs. 2 Ziff. 4 der Berufsordnung Niedersachsen vorgeschrieben, über die in Ausübung des Berufes gemachten Feststellungen und getroffenen Maßnahmen Aufzeichnungen zu fertigen (Berufsordnung Niedersachsen 1989). Gem. § 2 Abs. 5 Berufsordnung Hessen ist dem Tierarzt aufgegeben, über die in Ausübung seines Berufes gemachten wesentlichen Feststellungen und getroffenen Maßnahmen Aufzeichnungen zu fertigen (Berufsordnung Hessen 1987). Verletzungen dieser Standespflichten sind jedoch nur durch die tierärztlichen Berufsgerichte justitiabel, wobei allerdings auffällig ist, dass deren Urteile in Einzelfällen wesentlich härter ausfallen, als dies vor den sog. ordentlichen Gerichten denkbar wäre. Dabei sei insbesondere an Verurteilungen wegen Unterschreitung der gesetzlichen Mindestgebühren erinnert.

Nachdem sich standesrechtliche, teilweise gesetzliche und in jedem Fall vertragliche Pflichten des Tierarztes zur Dokumentation entwickelt haben, stellt sich dem Tierarzt natürlich die Frage, was er mit der Dokumentation zu veranlassen hat, nachdem er sie hergestellt hat. Insoweit steht außer Frage, dass der Tierarzt die Dokumentation zu archivieren hat. Es ist jedoch unklar, innerhalb welcher Fristen dies zu geschehen hat. Die Berufsordnungen der Tierärztekammern sehen teilweise keine und teilweise unterschiedlich lange Aufbewahrungsfristen vor. In Hessen beträgt die Aufbewahrungsfrist drei Jahre. In Bayern sind es sogar fünf Jahre. Gem. § 28 RöV sind die Originalbilder drei Jahre aufzubewahren. Danach ist eine Übertragung auf Datenträger gestattet. Ansonsten sind die Aufzeichnungen und die Röntgenbilder zehn Jahre zu verwahren. Sofern die Aufzeichnungen und Röntgenbilder im Rahmen einer Behandlung erstellt worden sind, beträgt die Aufbewahrungsfrist sogar dreißig Jahre (Hinrichs 1987). Die Fristen für die Aufbewahrung steverlich relevanter tiermedizinischer Dokumentationen betragen je nach Art der in $\S 147$ I AO genannten Unterlagen sechs oder zehn Jahre. So sind z. B. Rechnungen mit Buchfunktion zehn Jahre und ohne Buchfunktion sechs Jahre sowie Kontoauszüge mit Buchfunktion zehn Jahre aufzubewahren. Der Fristenlauf wird aber durch Betriebsprüfungen und weitere Tatbestände i. S. v. § 147 III S. 3 AO gehemmt, so dass er wesentlich länger sein kann (Tormöhlen 2004). Die Rechtsprechung hat sich bisher noch nicht zur Daver einer Aufbewahrungsfrist der Dokumentation als vertragliche Nebenpflicht des medizinischen Behandlungsvertrages geäußert. Es muss aber davon ausgegangen werden, dass der Auftraggeber vom Tierarz nach Ablauf der in den Berufsordnungen vorgegebenen Aufbewahrungsfristen keine Aufbewahrung derienigen Dokumentation verlangen kann, die nicht gesetzlichen Aufbewahrungs- fristen unterliegt. Denn insoweit sollte die Berufsordnung den Stand der gewissenhaften tiermedizinischen Handhabung widerspiegeln.

Der Tierarzt sollte aber prüfen, ob es überhaupt sinnvoll ist nach Ablauf der in den Berufsordnungen vorgesehenen Fristen diejenige Dokumentation zu vernichten, die keiner längeren gesetzlichen Aufbewahrungsfrist unterliegt. Denn die schriftliche Dokumentation kann dem Tierarzt aufgrund ihres Urkundscharakters ein wichtiges Beweismittel zu seiner eigenen Entlastung sein. Ebenso können technische Aufzeichnungen und insbesondere Röntgenbilder Gegenstände der richterlichen Augenscheinseinnahme oder der Sachverständigenbegutachtung sein und dem Tierarzt eine Hilfe in einem Haftpflichtrechtsstreit bieten. Deshalb macht es trotz des erheblichen Aufwandes durchaus Sinn, alle Dokumentationen solange aufzubewahren, bis die Veriährung der Schadenersatzansprüche eingetreten ist, die aus der dokumentierten Behandlung hergeleitet werden könnten (Kraft 1977; Borchert 1993). In der Literatur zur Haftung des Humanmediziners werden teilweise in Analogie zu § 28 RöV Aufbewahrungsfristen von zehn Jahren empfohlen (Matthies 1986).

Die Verjährungsfrist für Schadenersatzansprüche aus tierärztlichen Behandlungsverträgen, die vor dem 01.01.2002 geschlossen worden sind, betrug einheitlich 30 Jahre. Dabei konnte es im Ergebnis dahingestellt bleiben, ob der behauptete Anspruch aus einem reinen Dienstvertrag oder einem Kaufuntersuchungsvertrag, der von einem überwiegenden Teil der Rechtsprechung als Werkvertrag angesehen wird (BGH 1983; OLG München 1996; OLG Hamm 1997), abgeleitet wird. Denn Ansprüche wegen einer sog. positiven Vertragsverletzung des tierärztlichen Behandlungsvertrages unterlagen der 30jährigen Veriährung gem. § 195 BGB a. F. und der Kauf eines kranken Pferdes, dessen gesundheitliche Beeinträchtigungen von einem Tierarzł anlässlich einer Kaufuntersuchung schuldhaft nicht festgestellt und/oder nicht erwähnt wurden, stellte sich als sog. entfernter Mangelfolgeschaden dar, der nicht der kurzen Gewährleistungsfrist des Werkvertragsrechts gem. $\S 638$ BGB a. F. von sechs Monaten, sondern ebenfalls der dreißigiährigen Veriährung unterliegt. Für die noch nicht veriährten Schadenersatzansprüche aus vor dem 01.01.2002 geschlossenen Tierarztverträgen läuft ab dem 01.01.2002 gem. Art. $229 \S 6$ Abs. 4 EGBGB die Verjährungsfrist des BGB in der seit dem 01.01.2002 geltenden Fassung. Danach gilt ab dem Stichtag 01.01.2002 für die noch nicht verjährten Ansprüche aus Altverträgen sowie für die seit dem 01.01.2002 neu geschlossenen Behandlungsund Kaufuntersuchungsverträge, die mit dem Schuldrechtsmodernisierungsgesetz vom 26.11.2001 (BGBI. I S. 3138) eingeführte regelmäßige Verjährungsfrist von drei Jahren gem. $\S \S 195$ bs. 634 a I Ziff. 3, 195 BGB n. F.. Eine Ausnahme gilt für die Altverträge insweit, als dann die 30-jährige Veriährungsfrist weiterhin maßgeblich ist, wenn diese früher endet, als die ab 1.1.2002 neu zu laufen beginnende 30jährige verjährungsfrist. Es kann also nicht passieren, dass Schadensersatzansprüche aus einem Altvertrag durch die Schuldrechtsreform nun im Einzelfall einer 30-iährige Verfährungsfrist unterliegen könnten. Entgegen der teilweise vertretenen Ansicht unterliegen Schadenersatzansprüche aus tierärztlichen Kaufuntersuchungen nicht der zweijährigen Veriährungsfrist gem. $§ 634$ a I Ziff. 1; denn es handelt sich nach herrschender Meinung nicht um Arbeiten, die der Herstellung, 
Wartung und Veränderung des Tieres dienen (Sprau 2004). Diese recht kurze Frist von drei Jahren erhöht die Rechtssicherheit über die Fristen, innerhalb der ein Tierarzt eine Dokumentation, die keinen gesetzlichen Aufbewahrungsfristen unterliegt, zum Zweck seiner möglichen Rechtsverteidigung aufbewahren sollte, aber nur scheinbar (Adolphsen 2003). Denn die Veriährungsfrist beginnt gem. § 199 BGB n. F. mit dem Ende des Jahres zu laufen, in dem die schadenursächliche Behandlung oder Untersuchung stattgefunden hat und zur Entstehung des Anspruchs geführt hat und der Anspruchsteller von den anspruchsbegründenden Umständen und der Person des Schädigers Kenntnis erlangt hat oder sich in grob fahrlässiger Unkenntnis befand. Durch das subjektive Element der Schadenskenntnis in den neven Veriährungsregelungen wird zwar verhindert, dass Ansprüche veriähren, bevor ein Anspruchsteller Kenntnis von der Existenz seines Schadenersatzanspruchs erlangt hat, aber im Ergebnis kann diese Regelung wieder auf frühere Regelverjährung von dreiBig Jahren hinauslaufen. Denn Schadenersatzansprüche verjähren ohne Rücksicht auf ihre Kenntnis gem. § 199 III BGB n. F. in zehn Jahren ab Schadenentstehung oder in dreißig Jahren ab der schadensursächlichen Pflichtverletzung. Die kürzere Frist ist jeweils maßgeblich.

\section{Unterschiede zwischen den vertraglichen Dokumenta- tionspflichten des Tierarztes und des Arztes:}

Es war lange Zeit umstritten, ob die vorstehend erörterten Kriterien der humanmedizinischen Dokumentationspflicht auch für die Tiermedizin gelten und in welchem Umfang dies der Fall ist. Da die Dokumentation ein wesentlicher Bestandteil der Aufklärung des Patienten ist und aus dem vom grundrechtlich geschützten Selbstbestimmungsrecht abgeleiteten Informationsinteresse hergeleitet wird, war es nicht ohne weiteres selbstverständlich, auch dem Tierarzt eine Dokumentationspflicht aufzuerlegen. Dies gilt selbst dann, wenn es bereits seit langem einer standesrechtlichen Verpflichtung entsprach, eine Dokumentation zu führen. Das Standesrecht dient aber ausschließlich der Tierärzteschaft und nicht den auftraggebenden Tierhaltern. Ebenso entsprach es seit langem einem guten tiermedizinischen Brauch, eine Dokumentation zu führen. Letztendlich war dies für den häufig ambulant und außerhalb seiner Praxisräume behandelnden Tierarzt eine zwingende Notwendigkeit, um eine Gedächtnisstütze zu erlangen, anhand der er turnusmäßig seine Honorarabrechnungen erstellt. Trotz dieser Gesichtspunkte stellte die Rechtsprechung zunächst in Frage, ob eine Dokumentationspflicht für tiermedizinisches Handeln überhaupt anzunehmen ist (OLG Saarbrücken 1988).

Inzwischen hat das Tier in der Gesellschaft jedoch eine andere Stellung erfahren. Dies gilt nicht nur aufgrund der enorm gestiegenen Kaufpreise, die für einige Hochleistungspferde gezahlt werden. Vielmehr sind auch die Anforderungen an den Tierschutz gewachsen und es gilt eine andere Ethik im Umgang mit dem Tier und insbesondere dem Pferd. Dieser Verpflichtung muss ein Tierhalter gerecht werden, sodass es letztendlich nur eine Frage der Zeit war, bis eine Verpflichtung zur Dokumentation als vertragliche Nebenpflicht des tiermedizinischen Behandlungsvertrages von der Rechtsprechung bestätigt wurde. Inzwischen gilt es als tiermedizinischer Standard, die erhobenen Befunde zu dokumentieren und dabei insbesondere aufklärungspflichtige Umstände zu verzeichnen (OLG Stuttgart 1996; OLG Frankfurt 1999). Die Dokumenta- tion soll (nur) die wesentlichen Aspekte unter Außerachtlassung der Selbstverständlichkeiten in der Behandlung eines Pferdes umfassen, weil sich Grund und Anlass der Dokumentationspflicht des Tierarztes von der des Humanmediziners unterscheiden. Der ärztliche Behandlungsvertrag ist vom Grundrecht des Patienten auf Selbstbestimmung beherrscht, während dies im tierärztlichen Behandlungsvertrag keine Rolle spielt, sondern die wirtschaftliche Bedeutung des Tieres sowie die rechtlichen und sittlichen Gebote des Tierschutzes zu beachten sind (BGH 1977). Deshalb ist als Dokumentationsmaßstab eine stichwortartige Aufzeichnung ausreichend, die einen anderen Tierarzt in die Lage versetzt, die Behandlung weiter zu führen, ohne Irrtümern zu unterliegen (OLG Hamm 2003). An Art und Umfang einer Dokumentation dürfen jedoch nicht so hohe Anforderungen wie in der Humanmedizin gestellt werden (LG Verden 2004). Das ist insbesondere auch aus tatsächlichen Gründen richtig; denn ein Großteil aller tiermedizinischen Behandlungen am Pferd wird von einem praktischen Tierarzt ohne Helfer in einem Stall oder auf der Weide durchgeführt. Die Umweltbedingungen unterscheiden sich also schon ganz erheblich von der ambulanten humanmedizinischen Behandlung, die regelmäßig in einer Arztpraxis stattfindet. Dort ist das Aufzeichnen wesentlich einfacher als unter den Behandlungsbedingungen eines Tierarztes.

Die Ant der Dokumentation ist ohnehin sowohl in der Veterinär- als auch der Humanmedizin maßgeblich durch ihren medizinischen Sinn bestimmt. Die Dokumentation erfolgt zwar auch im Interesse des Patienten. Sie ist jedoch zunächst für die ärztliche Behandlung eine Hilfe und dient der Erbringung ärztlicher Leistungen (Bender 1997). Deshalb ist eine patientenverständliche Form der Dokumentation nicht geschuldet. Vielmehr muss die Dokumentation für einen anderen Mediziner verständlich sein (BGH 1983 b, BGH 1984). Dabei kann auch eine Dokumentation in Stichworten und Symbolen genügen, solange der nachbehandelnde Mediziner sie verstehen kann. Sie muss so ausführlich sein, dass ein Nachbehandler aus der Dokumentation heraus ein Bild vom bisherigen Krankheitsverlauf, der Diagnose oder den erhobenen Befunden und der Therapie gewinnt. Ebenso sollen die Prognose oder Behandlungsempfehlung aus der Dokumentation hervorgehen. Das Letzte muss dringend zu empfehlender Bestandteil der Dokumentation sein, wenn sich der Auftraggeber beratungsresistent verhält z. B. eine Überweisung in eine stationäre Klinikbehandlung ablehnt. Dabei kann es sich in Einzelfällen als Vorteil erweisen, die Aufklärung vom Auftraggeber gegenzeichnen zu lassen, um die Vermutung der Vollständigkeit und Richtigkeit der Urkunde i. S. v. § 416 ZPO zu erlangen und das Gericht zu zwingen, den Tierarzt gem. § 448 ZPO von Amts wegen als Partei über die dokumentierte Maßnahme zu vernehmen, falls der Auftraggeber sie in Abrede nehmen sollte (OLG München 1988). Ohnehin gilt der Grundsatz, dass Besonderheiten - gleich welcher Art - dokumentiert werden müssen (Stöhr 2003).

\section{Folgen einer Dokumentationspflichtverletzung:}

Die Dokumentationspflichtverletzung führt in jedem Fall zu einem Korrekturanspruch des Patienten. Dieser kann vom Arzt verlangen, dass er seine Dokumentation ergänzł und/oder berichtigt. Darüber hinaus wird dem Patienten regelmäßig ein Zurückbehaltungsrecht am Honorar eingeräumt, bis der Arz† seiner Dokumentationspflicht nachkommt (Wasserburg 1980; 
Kramer 1998). Dies gilt allerdings nur solange noch die Möglichkeit zur ordnungsgemäßen Dokumentation besteht. Eine Schadenersatzverpflichtung des Arztes wird jedoch im Gegensatz zur Aufklärungspflichtverletzung aus der Dokumentationspflichtverletzung nicht hergeleitet (BGH 1983 c; BGH 1988). Vielmehr sind die Folgen einer Dokumentationspflichtverletzung prozessualer Natur. Der Arzt, der einem Antrag auf Vorlegung seiner Dokumentation gemäß $\S 421$ ZPO nicht nachkommt, erleidet die bereits in Kapitel II erläuterten prozessualen Nachteile. Das Gericht kann im Wege einer prozessrechtlichen Sanktion das Nichtbefolgen einer richterlichen Anordnung bestrafen und die Behauptungen der beweispflichtigen Partei als bewiesen ansehen.

Die beweisrechtliche Konsequenz einer Dokumentationspflichtverletzung darf ansonsten nicht generell eine Beweislastumkehr, sondern nur eine Beweiserleichterung bewirken, die sich bis zur Beweislastumkehr in den Bereichen entwickeln kann, die es dem Patienten unzumutbar machen, angesichts der Dokumentationsversäumnisse die volle Beweislast zu tragen (BGH 1978). Die unterbliebene Dokumentation ist eine fahrlässige vorprozessuale Beweisvereitelung und löst die Fiktion aus, dass die nicht dokumentierte Behandlung oder Maßnahme nicht durchgeführt worden ist. Diese Fiktion wird durch eine Analogie zu den prozessualen Vorschriften über die Folgen einer Nichtvorlegung von Urkunden (§ 427 ZPO) sowie einer Urkundenbeseitigung ( $\S 444$ ZPO) und der Verweigerung einer Parteivernehmung (§ 446 ZPO) hergestellt (Stürner 1979). Die Fiktion ist für den dokumentationssäumigen Arz† widerlegbar, wobei sich die Anforderungen an die Gegenbeweisführung nach der Schwere der Dokumentationspflichtverletzung und ihrer Bedeutung für die Beweisführung richten (Matthies 1986). Sofern das Unterbleiben der noch nicht dokumentierten Behandlung einen groben Kunstfehler darstellt, wird aus der Beweiserleichterung eine Beweislastumkehr zu Lasten des dokumentationspflichtigen Arztes. Ein grober Fehler im juristischen Sinne liegt dann vor, wenn der Fehler bei objektiver Betrachtung nicht unterlaufen darf und unverständlich sowie unverantwortlich erscheint, weil ein Verstoß gegen bewährte tierärztliche Behandlungsregeln oder gesicherte medizinische Erkenntnisse vorliegt (BGH 1983 d).

Diese Rechtsgrundsätze werden nahezu vollständig auf den tiermedizinischen Bereich übertragen. Auch dort wird in der Literatur die Auffassung vertreten, dass die Dokumentationspflichtverletzung nicht zum selbstständigen Schadenersatzanspruch des Tiereigentümers führt (Deipenbrock 1991). Dies dürfte aber in der Tierarzthaftung falsch sein; denn die Dokumentationspflichtverletzung kann insbesondere bei fehlerhafter Befundbeschreibung zu Vermögensdispositionen des Tiereigentümers führen. Eine kunstfehlerhafte Protokollierung der anlässlich einer Kaufuntersuchung erhobenen Befunde kann dazu führen, dass ein Verkäufer sein Pferd weit unter Wert verkauft oder gar das Pferd töten lässt (LG Hannover 1987). Ebenso können dem Käufer Schäden entstehen, wenn der Tierarzt in kunstfehlerhafter Weise die Dokumentation von erwähnungspflichtigen Befunden unterlässt und der Käufer in Kenntnis dieser Befunde das Pferd nicht oder nicht zu dem gezahlten Preis erworben hätte.

Das Zurückbehaltungsrecht am Honorar wird in der Rechtsprechung ebenfalls noch kontrovers behandelt. Im Arztrecht wird im Falle einer unzureichenden oder unklaren Dokumentation einhellig ein Zurückbehaltungsrecht angenommen.
Dies gilt jedenfalls solange die Erbringung einer pflichtgemäBen Dokumentationsleistung möglich erscheint (Roth 1979; Wasserburg 1980; Ullrich 1984). Im Tierarztrecht gibt es kein einheitliches Meinungsbild. Häufig wird ein solches Zurückbehaltungsrecht abgelehnt (AG Hameln 2003; a. A. AG Burgwedel 2004). Dafür fehlt aber ein dogmatischer Ansatzpunkt; denn es steht im Dienstvertragsrecht außer Frage, dass der dienstverpflichtete Tierarzt gem. § 614 BGB vorleistungspflichtig ist. Hat er seine Dienstleistung noch nicht erbracht, weil er der Verpflichtung zur Dokumentation nicht oder nur unzureichend nachgekommen ist und deshalb Auskunftsbedarf des Auftraggebers besteht, ist das Honorar noch nicht zur Zahlung fällig. Letztendlich ist es auch dogmatisch nicht zu rechtfertigen, die Dokumentationspflicht des Tierarztes von der des Arztes im Hinblick auf die Honorarfälligkeit unterschiedlich zu behandeln; denn insoweit unterscheiden sich die ärztlichen Pflichten nicht von den tierärztlichen. Gänzlich anders ist lediglich die beendete, aber schlecht erfüllte tierärztliche und ärztliche Dienstleistung zu behandeln. Insoweit ist das Honorar fällig und trotz Schlechtleistung zu zahlen. Der Tiereigentümer ist auf den Schadenersatzanspruch zu verweisen und muss ggfs. gegenüber dem tierärztlichen Honoraranspruch aufrechnen. Für eine in der Literatur geforderte und in der Rechtsprechung vereinzelt ausgeurteilte Freistellung von der Honorarzahlungspflicht gibt es keine gesetzliche Grundlage (Bemmann 2004).

Ansonsten besteht hinsichtlich der Folgen einer Dokumentationspflichtverletzung völlige Übereinstimmung der Tierarzthaftung mit der Arzthaftung. Insbesondere gilt die Vermutung, dass eine nicht dokumentierte, aufzeichnungspflichtige Maßnahme nicht durchgeführt worden ist (OLG Stuttgart 1996). Dann trifft den Tierarzt die Beweislast, dass die Maßnahme doch durchgeführt wurde (OLG Frankfurt 1999).

\section{Das Einsichtsrecht in die Dokumentation:}

Genauso wie die Dokumentation als vertragliche Nebenpflicht des ärztlichen Behandlungsvertrages erst sukzessive entwickelt wurde, bestand ein Einsichtsrecht in ärztliche Behandlungsunterlagen zunächst nicht und später in eingeschränktem Ausmaß. Der Grund liegt in dem Urkundscharakter der medizinischen Behandlungsunterlagen. Ein Einsichtsrecht an Urkunden im Sinne von $\S \S 810,811$ BGB besteht nur dann, wenn die Urkunde auch im Interesse des Patienten erstellt worden ist. Dieses Interesse des Patienten wurde anfänglich aus den bereits dargestellten Gründen abgelehnt. Die Dokumentation sollte nämlich nur eine bloße Gedächtnisstütze des Arztes sein, dem die Entscheidung obliegt, ob er überhaupt dokumentiert und wie vollständig und sorgfältig er die Krankengeschichte aufzeichnet. Die persönlichen Aufzeichnungen des Arztes sollten zugleich auch schützenswerte Persönlichkeitsrechte des Arztes beinhalten. Deshalb wurde lange Zeit ein Einsichtsrecht abgelehnt (so letztmalig OLG Celle 1978). Ein Einsichtsrecht sollte allenfalls dann in Betracht kommen, wenn bereits plausible Gründe für einen Behandlungsfehler und daraus resultierende Ersatzansprüche des Patienten sprechen. In solchen Fällen sollte es nach Treu und Glauben gemäß § 242 BGB angemessen sein, dem Patienten ein Einsichtsrecht zu gewähren (Stürner 1979).

Spätestens seit der grundlegenden Entscheidung des BGH (BGH 1978 a) ist diese Auffassung überholt. Seitdem ent- 
spricht es einhelliger Rechtsprechung, dass die ärztlichen Krankenunterlagen keine internen Gedächtnisstützen des Arztes, sondern auch im Interesse des Patienten erstellte Urkunden sind, die im Rahmen einer selbstständigen Nebenpflicht des ärztlichen Behandlungsvertrages herzustellen sind. Die Rechtsprechung des BGH wird u. a. von dem Gedanken getragen, dass die Ethik eines ärztlichen Behandlungsvertrages es erfordert, an die ärztliche Tätigkeit mindestens genauso hohe Sorgfaltsmaßstäbe wie an den Vermögens- oder Hausverwalter zu stellen. Dieser geht nur mit Sachen um und ist gleichwohl verpflichtet, seinem Auftraggeber Rechenschaft abzulegen. Deshalb erfordert der Umgang mit Lebewesen erst recht eine Pflicht zur Rechenschaft gegenüber dem Patienten. Dies rechtfertigt sich insbesondere auch durch den Vergleich des Arztes mit anderen akademischen Dienstleistern. Der Rechtsanwalt ist gegenüber dem Mandanten nicht nur zur vollständigen Akteneinsicht, sondern auch zum Hinweis auf die konkret im Einzelfall gegen ihn bestehende Regressmöglichkeit und die Veriährung des Regressanspruchs verpflichtet. Anderenfalls handelt er kunstfehlerhaft. Deshalb entspricht es nun ständiger Rechtsprechung, dem Patienten gegen den behandelnden Arzt einen Anspruch auf Einsicht in die ärztlichen Krankenunterlagen zuzubilligen. Dabei werden unterschiedliche dogmatische Ansätze gewählt. Ein Anspruch auf Einsicht in die schriftlichen Unterlagen des Arztes ergibt sich aus $\S \S 810,811$ BGB, weil es sich bei diesen Schriftstücken um Urkunden im Sinne des Gesetzes handelt und diese auch im Interesse des Patienten erstellt worden sind. Damit wäre das Einsichtsrecht des Patienten aber auf die schriftlichen Teile der ärztlichen Dokumentation beschränkt. Technische Aufzeichnungen und insbesondere Röntgenbilder wären ausgenommen. Deshalb wird das Einsichtsrecht außerdem auch als selbstständiger nebenvertraglicher Anspruch aus dem ärztlichen Behandlungsvertrag angesehen, weil es im Lichte von § 242 BGB treuwidrig wäre, dem Patienten eine seinen Grundrechten zuwider laufende Statistenrolle im Rahmen des ärztlichen Behandlungsvertrages einzuräumen (LG Göttingen 1979). Um dem Einsichtsrecht voll umfänglich zu entsprechen, wird seit der Grundsatzentscheidung des BGH regelmäßig der Einsichtsanspruch als nebenvertraglicher Anspruch aus dem Behandlungsvertrag (LG Limburg 1979) oder als Anspruch des Patienten auf Rechenschaftslegung gegen seinen Arzt begründet (OLG Bremen 1980).

Allerdings bleibt es auch nach dem Wandel in der Rechtsprechung uneingeschränkt beim Eigentums- und Urheberrecht des Arztes. Deshalb kommt kein Herausgabeanspruch des Patienten, sondern nur ein Einsichtsanspruch in Betracht (Ahrens 1979). Außerdem hat der Arzt das sog. „therapeutische Privileg". Er kann das Einsichtsrecht des Patienten einschränken, wenn der Heilungserfolg durch die Ausübung des Einsichtsrechts gefährdet würde. Dies wäre z. B. der Fall, wenn nach Ausübung des Einsichtsrechts eine Suizidgefahr bestände. Allerdings soll die Einschränkung nur in der Weise erfolgen, dass dem Patienten nicht persönlich, sondern seinem Betrever, einem anderen Arzt oder einer ähnlichen Person die Einsicht gewährt wird (Wasserburg 1980; LG Saarbrücken 1996; Kern 1996).

In der Tiermedizin wurde eine Einsicht in die Dokumentation trotz der grundlegenden Entscheidung des BGH zum Einsichtsrecht in humanmedizinische Unterlagen zunächst fälschlicherweise nicht als selbstständig einklagbarer Anspruch angesehen (Deipenbrock 1991). Es war lediglich unstreitig, dass auch im Tierarzthaftungsrechtsstreit die Urkundsvorlage aufgrund gerichtlicher Anordnung im Prozess zu erfolgen hat. Ansonsten wurde lediglich empfohlen, Einsicht zu gewähren. Die Gründe für die abweichende Haltung der Tiermedizin lagen auf der Hand; denn das Selbstbestimmungsrecht des Patienten spielt im tiermedizinischen Behandlungsvertrag keine Rolle. Vielmehr steht die wirtschaftliche Bedeutung des Tieres im Vordergrund und es sind (nur) die rechtlichen und sittlichen Gebote des Tierschutzes zu beachten (BGH 1977). Gleichwohl gibt es mehrere tragende Elemente des tierärztlichen Behandlungsvertrages, die ein Einsichtsrecht in die tierärztliche Dokumentation rechtfertigen. So ist immerhin auch der auftraggebende Tierhalter ein Mensch und dies gebietet es, ihm in keinem schuldrechtlichen Vertrag die Rolle eines Statisten aufzuerlegen. Auch der Tierarzt geht mit schützenswertem Leben um, so dass er sich ebenso einen Vergleich mit dem vollumfänglich rechenschaftspflichtigen Vermögensverwalter gefallen lassen muss. Außerdem hat der Tierhalter die Verpflichtung, die ethischen Grundsätze der Tierhaltung sowie die Interessen des Tierschutzes zu überwachen. Dies kann es gebieten, ihm auch außerhalb eines Tierarzthaftpflichtrechtsstreits ein Informationsinteresse zuzubilligen. Dies gilt umso mehr, als der Urkundscharakter der schriftlichen Bestandteile der tiermedizinischen Dokumentation ebenso außer Frage steht wie in der humanmedizinischen Dokumentation. Letztendlich muss auch in der Tierarzthaftung der Grundsatz der Waffengleichheit gelten. Deshalb muss es auch dem auftraggebenden Tiereigentümer möglich sein, vor der Entscheidung über die Führung eines Schadenersatzprozesses die Krankenunterlagen zu Informationszwecken einzusehen und ggf. sachverständig prüfen zu lassen.

Diese Denkansätze gebieten m. E. ein Einsichtsrecht in die tierärztliche Dokumentation. Um hierüber Rechtssicherheit zu erlangen, habe ich im Jahre 1990 und 1991 jeweils einen "Pilotprozess" vor dem Landgericht Kassel und dem Landgericht Hildesheim rechtshängig gemacht. Dort habe ich für einen Pferdeeigentümer die Einsichtnahme in die tiermedizinische Dokumentation in der Weise eingeklagt, dass der Tierarzł auf Kosten des Pferdeeigentümers vollständige Kopien der Krankenunterlagen fertigt und übersendet. Das Landgericht Kassel hat festgestellt, dass ein Tierarzt verpflichtet ist, Behandlungsunterlagen zu führen und es sich hierbei um Urkunden im Sinne von $\S 810$ BGB handelt, die auch im Interesse des Pferdeeigentümers erstellt worden sind. Allein daraus folge ein Einsichtsrecht gem. $\S \S 810,811$ BGB, welches der Tiereigentümer nicht am Aufbewahrungsort der Urkunde in der tierärztlichen Praxis ausüben müsse. Vielmehr könne er die Herstellung und Übersendung von Kopien gegen Kostenübernahme verlangen, um in Ruhe und ggf. unter sachverständiger Beratung die Unterlagen einzusehen (LG Kassel 1990). Der Tierarzt hat das Interesse des Pferdeeigentümers an den Krankenunterlagen abgelehnt und diese als seine persönliche Gedächtnisstütze bezeichnet. Er hat deshalb Berufung zum OLG Frankfurt/Main eingelegt. In zweiter Instanz ist das Einsichtsrecht mit der Begründung bestätigt worden, dass auch die tiermedizinische Dokumentation keine bloße Gedächtnisstütze des Tierarztes ist, sondern dem Pferdeeigentümer zur Entscheidung über eine Weiterbehandlung des Tieres oder auch selbstständigen Prüfung von Schadenersatzansprüchen dienen soll. Dabei müsse der Tierarzł auch die Gefahr einer Selbstbezichtigung hinnehmen, weil nur so im Tierarzthaftungsprozess dem Grundsatz der Waffengleichheit 
Rechnung getragen werden könne. Anderenfalls habe der Tierarzt im Vergleich zum Pferdeeigentümer einen ähnlich groBen Wissensvorsprung wie der Arzł gegenüber seinem Patienten. Deshalb folge der Anspruch auf Einsichtsgewährung aus $\S \S 810,811$ i. V. m. 242 BGB (OLG Frankfurt 1991).

Das Landgericht Hildesheim hat sich ausdrücklich damit auseinandergesetzt, dass im tierärztlichen Behandlungsvertrag das ein Einsichtsrecht in ärztliche Behandlungsunterlagen rechtfertigende Selbstbestimmungsrecht des Patienten keine Rolle spielt. Es hat aber hervorgehoben, dass dem veränderten Stellenwert des Tieres, den dieses spätestens seit Inkrafttreten des Gesetzes zur Verbesserung der Rechtsstellung des Tieres im bürgerlichen Recht vom 20.08.1990 (BGBI. I 1990, S. 1762) erlangt hat, auch in der Medizin Rechnung getragen werden müsse. Dabei spiele die besondere Verantwortung des Tierhalters für seine Mitgeschöpfe eine Rolle. Insbesondere deshalb sei ein Interesse des Tierhalters an der Erstellung einer tiermedizinischen Dokumentation nicht mehr wegzudiskutieren. Außerdem sei das Interesse einer Beweissicherung und einer Grundlage für die Entscheidung über weitere oder erneute Behandlungen durch einen anderen Tierarzt das gleiche, wie in der Humanmedizin. Letztendlich kämen in der Tiermedizin Einschränkungen des Einsichtsanspruchs durch das therapeutische Privileg im Gegensatz zur Humanmedizin auch nicht einmal denktheoretisch in Betracht. Deshalb bestehe ein Anspruch gem. $\S \S 810,811,611,242$ BGB auf Übersendung von Kopien der vollständigen Krankenunterlagen gegen Kostenübernahme zum selbstständigen Studium (LG Hildesheim 1992). Gegen dieses Urteil ist kein Rechtsmittel eingelegt worden. Seitdem entspricht es ständiger Rechtsprechung, dass auch gegen den behandelnden Tierarz ein selbstständig einklagbarer Anspruch auf Einsicht in die tiermedizinische Dokumentation besteht. Dies gilt auch für die Einsicht in Röntgenbilder. Der Pferdeeigentümer kann auf seine Kosten die Anfertigung und Übersendung von Kopien oder aber die Einsicht durch vorübergehende Überlassung der Röntgenbilder an einen anderen Tierarzt im Sinne von $\S 28$ Abs. 6 RöV verlangen (AG Limburg 2003).

\section{Andere Rechte und Rechte Dritter an der Dokumenta- tion}

Die modernen Tierarztpraxen und insbesondere Kliniken arbeiten mit EDV. Dadurch unterliegen sie den Vorschriften des Bundesdatenschutzgesetzes in der Fassung vom 14.01.2003 (BGBI. I S. 66). Deshalb sind die personenbezogenen Daten des auftraggebenden Tierhalters nur mit seiner Einwilligung zu speichern. Diese kann bei Kliniken zweckmäBigerweise formularmäßig in dem Aufnahmeschein erteilt werden. An den Daten kann der Tierhalter sein Auskunftsrecht gem. § 19 BDSG geltend machen oder gem. § 20 BDSG die Daten berichtigen, löschen, sperren lassen oder ihnen widersprechen, sofern er sie für unrichtig hält.

Die Dokumentation über die Behandlung des Patienten unterliegt den durch $\S 203$ । Ziff. 1 StGB geschützten Privatgeheimnissen. Dies gilt für Arzt und Tierarzt gleichermaßen. Die Weitergabe der Dokumentation an ärztliche Verrechnungsstellen zum Zweck der Rechnungserstellung und Abtretung des Honorars zum Zweck des Einzuges ist deshalb wegen Verstoßes gegen ein gesetzliches Verbot gem. § 134 BGB nichtig, solange der Patient nicht zustimmt (BGH 1991). Dies gilt nicht für die tierärztliche Abrechnung. Insoweit gilt zwar auch für den Tierarzł grundsätzlich die Strafbarkeit der Weitergabe von Geheimnissen des Auftraggebers. Die in der tiermedizinischen Dokumentation verzeichneten Behandlungsdaten und -maßnahmen enthalten aber typischerweise keine Privatgeheimnisse des Auftraggebers über seine gesundheitliche Verfassung, sondern Informationen über das behandelte Tier. Dem Patienten "Tier" kommt jedoch der strafrechtliche Geheimnisschutz nicht zugute, so dass die Abtretung zur Einziehung tierärztlicher Honorarforderungen an tierärztliche Verrechnungsstellen wirksam ist und keiner Zustimmung des Tiereigentümers bedarf (LG Lüneburg 1993, OLG Celle 1995). Die vereinzelt vertretene gegenteilige Rechtsauffassung hat sich nicht durchgesetzt (LG Bochum 1993).

Außerdem bestehen Auskunfts- und Einsichtsrechte der Sozialversicherungsträger an der ärztlichen Dokumentation aus übergegangenem Recht gem. §§ 401, 412 BGB, 116 SGB X. Insbesondere bei vermutetem Behandlungsfehler steht den gesetzlichen Krankenkassen ein Einsichtsrecht in die ärztliche Dokumentation zu (Hüpers 1994; Wessel 2002). Diese Rechtsgedanken dürften auf die tierärztlichen Dokumentationen ebenso zugunsten eines Tierversicherers Anwendung finden, auf den Ersatz- oder Gewährleistungsansprüche des Tiereigentümers gem. $\S \S 67,118$ WG übergegangen sind.

Letztendlich werden die Rechte des Patienten sowie des Tierhalters an der Dokumentation eingeschränkt, wenn gegen den Arzt oder Tierarzt strafrechtliche Ermittlungen z. B. wegen Abrechnungsbetruges zu führen sind. Dabei dürfen aber dem Geheimnis gem. § 203 । Ziff. 1 StGB unterliegende Informationen nur für strafrechtliche Zwecke genutzt werden, soweit dies unerlässlich ist. Nur insoweit haben Belange des Gemeinwohls wie der staatliche Strafanspruch und das Rechtsstaatsprinzip den Vorrang gegenüber den Patientenrechten (Rudolf 1991).

\section{Fazit}

Nach diesem Überblick lässt sich folgendes Ergebnis festhalten:

1. Die tiermedizinische Behandlung unterliegt der Dokumentationspflicht.

2. Die Dokumentation wird als selbständige vertragliche Nebenpflicht des tierärztlichen Behandlungsvertrages geschuldet und auch im Interesse des Pferdeeigentümers erstellt.

3. Die Anforderungen an Art und Umfang der Dokumentation sind in der Tiermedizin geringer als in der Humanmedizin.

4. Die prozessualen Folgen einer tiermedizinischen Dokumentationspflichtverletzung sind die gleichen wie in der Humanmedizin. Sie können von Beweiserleichterungen bis hin zur Beweislastumkehr reichen.

5. Zusätzlich kommen materiellrechtliche Folgen einer tiermedizinischen Dokumentationspflichtverletzung in Betracht. Macht der Tiereigentümer aufgrund einer vom Tierarzt zu vertretenden fehlerhaften Dokumentation nachteilige Vermögensdispositionen geltend, kann ihm ein Anspruch aus positiver Vertragsverletzung in Höhe des eingetretenen Vermögensschadens zustehen.

6. Es besteht ein selbstständig einklagbarer Anspruch auf Einsicht in die tiermedizinische Dokumentation. 


\section{Abkürzungen}

a. A. anderer Ansicht

a. F. alte Fassung des BGB bis 31.12.2001

AG Amtsgericht

AgrarR Agrar- und Umweltrecht, Zeitschrift für das gesamte Recht der Landwirtschaft, der Agrarmärkte und des ländlichen Raumes

AO Abgabenordnung

Art. Artikel

ArztR Arztrecht (Zeitschrift)

BDSG Bundesdatenschutzgesetz

BGB Bürgerliches Gesetzbuch

$\mathrm{BGBI}$ Bundesgesetzblatt, Band, Jahrgang und Seite

BGHZ Entscheidungen des Bundesgerichtshofes in Zivilsa

chen, Band u. Seite

BO Berufsordnung der Tierärztekammer

BVerfG Bundesverfassungsgericht und zugleich auch Ent scheidungen des Bundesverfassungsgerichts (Band und Seite)

CR Computer und Recht (Zeitschrift)

EGBGB Einführungsgesetz zum Bürgerlichen Gesetzbuch

GG Grundgesetz

LG Landgericht

MedR Medizinrecht, Zeitschrift

n. F. neve Fassung des BGB ab 01.01.2002

NJW Neve Juristische Wochenzeitschrift (Zeitschrift)

NJW-RR Neue Juristische Wochenschrift-Rechtsprechungsre port Zivilrecht

OLGR OLG-Report, Schnelldienst zur Zivilrechtsprechung der Oberlandesgerichte (Zeitschrift)

RöV Röntgenverordnung vom 8. Januar 1987 BGBI I S. 114

SGB Sozialgesetzbuch I. - XI. Buch

StGB Strafgesetzbuch

StPO Strafprozessordnung

StrlSchV Strahlenschutzverordnung vom 20. Juli 2001 BGBI.

IS. 1714

SVT Sozialversicherungsträger

VersR Zeitschrift für Versicherungsrecht, Haftungs- und

Schadensrecht

WG Versicherungsvertragsgesetz

ZfS Zeitschrift für Schadensrecht

ZPO Zivilprozessordnung

\section{Literatur}

Adolphsen J. (2003): Haftungsrechtliche Aspekte der veterinärmedizinischen Kaufuntersuchung von Pferden, VersR, 1088-1091

AG Burgwedel (2004): AgrarR 110

AG Hameln (2003): Urteil vom 10.12.2003 Az.: 32 C 144/03

AG Limburg (2003): Urteil vom 23.09.2003 Az.: 4 C 995/03

Ahrens H.-J. (1979): Anmerkung zum Urteil LG Göttingen v.

16.11.1978 Az.: $2 \bigcirc 152 / 78$, NJW, 602-603

Bemmann K. (2004): Die tierärztliche Aufklärungspflicht, AgrarR, 107-111 [109]

Bender A. (1997): Der Umfang der ärztlichen Dokumentationspflicht, VersR, 918-928

Berufsordnung Hessen (1987): DTBI, $112 \mathrm{ff}$

Berufsordnung Niedersachsen (1989): DTBI, $748 \mathrm{ff}$.

BGH (1952): NJW, $661-662$

BGH (1972): NJW 1520-1521 = VersR 887-889

BGH (1975): NJW 1463-1465

BGH (1977): NJW 1102-1103 = DpT 754

BGH (1978 a): NJW 2337-2339= VersR 1022-1024

BGH (1978 b): NJW 1681-1682

BGH (1983 a): NJW 2078-2080 = BGHZ 87, 23-246

BGH (1983 b): VersR 983-984

BGH (1983 c): NJW 328-330

BGH (1983 d): NJW 2080-2082

BGH (1984): NJW 1403-1405 = VersR 386-

BGH (1988): NJW 2949-2951

BGH (1991): NJW 2955-2958

Borchert G. (1993): Dokumentation des Arztes, CR 718-723

BVerfG (1979): Bd. 52131 ff. [152]
Deipenbrock R. (1991): Umfang und Risiken der tierärztlichen Dokumentationspflicht, DpT, 670-675

Franzki H. und Franzki D. (1975): Waffengleichheit im Arzthaftungsprozess, NJW, 2225-2229

Hinrichs O. (1987): Die neue Röntgenverordnung, NJW 2284-2285

Hüpers F. (1994): Auskunftsansprüche des SVT nach dem UmweltHG? VersR, 653-656

Jäckel H.-O. (1971): Über den Begriff, die Pflicht zur Führung und Aufbewahrung sowie die Rechtsnatur der Krankenunterlagen in: Die juristische Problematik in der Medizin Bd. II, 163-182

Kern B.-R. (1996): Anmerkung zum Urteil des LG Saarbrücken vom 20.09.1995 Az: 16 S 1/93, MedR, 324

Kraft H. (1977): Forensische Probleme der Ankaufsuntersuchung. Über die Rechtsnatur; die Pflicht zur Führung und Aufbewahrung der Patientenunterlagen; die tierärztliche Bescheinigung, DpT, 206-209 Kramer W. (1998): Keine Vergütungspflicht bei fehlendem Interesse an der Dienstleistung infolge Schlechterfüllung, MDR, 324-332

LG Bochum (1993): NJW 1535-1537

LG Göttingen (1979): NJW 601-602

LG Hannover (1987): Urteil v. 14.04.1987 Az.: 19 ○ 36/87

LG Hildesheim (1992): NJW-RR 415-416

LG Kassel (1990): Urteil vom 15.08.1990 az.: $6 \bigcirc 1270 / 90$

LG Limburg (1979): NJW 607-608

LG Lüneburg (1993): NJW 2994

LG Saarbrücken (1996): MedR 323-324

Matthies K.-H. (1986): Zu Inhalt und Umfang der ärztlichen Dokumentationspflicht und den Rechtsfolgen einer ungenügenden Dokumentation (Beweisvereitelung), JZ, 959-962

Mehrhoff F. (1990): Aktuelles zum Recht der Patientendokumentation, NJW, 1524-1525

OLG Bremen (1980): NJW 644

OLG Celle (1978): NJW 1200

OLG Celle (1995): NJW 786

OLG Frankfurt (1991): Urteil vom 20.09.1991 Az.: 25 U 263/90

OLG Frankfurt (1999): AgrarR 218-219

OLG Hamm (1997): OLGR 191-192

OLG Hamm (2003): VersR $1139-1140$

OLG München (1988): VersR 1136-1137

OLG München (1996): VersR 731-732

OLG Saarbrücken (1988): VersR 850-85

OLG Stuttgart (1996): VersR 1029-1030

Roth W.-H. (1979): Der Vergütungsanspruch bei schlechter Leistung im Recht der freien Berufe; Das Recht zur Verweigerung der Vergütung, VersR, 494-504

Rudolf W. (1991): Rechte des Patienten im Ermittlungsverfahren gegen seinen Arzt wegen Abrechnungsbetruges, NJW, 2337-2338

Schüle E. (2003): Der Tierarzt als Korrektiv im Pferdesport - Reiter, Halter und Nutzer aus der Sicht der Tiermedizin, Pferdeheilkunde $19,397-403$

Sprau H. (2004): in: Paland O. (Hrsg.) Bürgerliches Gesetzbuch, Kommentar, 63. Aufl., § 634 a Rz 8, 12

Stöhr K. (2003): Wie ausführlich muss die Patientenaufklärung dokumentiert werden? Patientenrechte, 65-68

Strunz W. (1990): Aufbewahrungsfristen für Unterlagen bei Ärzten, ArztR, $210-213$

Stürner R. (1979): Entwicklungstendenzen des zivilprozessualen Beweisrechts und Arzthaftungsprozess, NJW, 1225-1230

Tormöhlen H. (2004): in: Papperitz G. (Hrsg.) ABC Betriebsprüfung, Kommentar, 4. Aufl.

Ullrich H. (1984): Lohngewähr oder Mängelgewährleistung. Zum Lohnanspruch bei unsorgfältiger Dienstleistung, NJW, 585-589

Wasserburg K. (1980): Die ärztliche Dokumentationspflicht im Interesse des Patienten, NJW, 617-624

Wessel M. (2002): Der Anspruch auf Einsichtnahme in die Patientendokumentation durch gesetzl. Krankenkassen bei vermutetem Behandlungsfehler, ZfS, 461-462

Wiethaupt R. (1954): Herausgabe von Krankenblättern an Dritte? JR, 154-155

Kai Bemmann

Rechtsanwalt, Öffentlich bestellter und vereidigter Sachverständiger für Pferdezucht und -haltung

Andreaswall 2, 27283 Verden/Aller

bemmann@bernerfischer-partner.de 